\title{
Positive reform of tuna farm diving in South Australia in response to government intervention
}

\author{
P Whyte, D J Doolette, D F Gorman, D S Craig
}

Department of

Anaesthesia and Intensive Care, Royal Adelaide Hospital, Adelaide, Australia P Whyte

D F Gorman

Department of Anaesthesia and Intensive Care, The

University of Adelaide, Adelaide, Australia 5005

D J Doolette

Department of Medicine, University of Auckland, Auckland, Australia D F Gorman

Workplace Services, Department for Administrative and Information Services, Government of South Australia, Adelaide,

Australia

D S Craig

Correspondence to: Dr D Doolette David.Doolette@adelaide .edu.au

Accepted 12 September 2000

\begin{abstract}
Objectives-Much of the tuna harvested in South Australia since 1990 has involved "farming" techniques requiring the use of divers. From 1993 to 1995,17 divers from this industry were treated for decompression illness (DCI). In response, the State Government introduced corrective strategies. A decrease in the number of divers presenting for treatment was subsequently recorded. Consequently, the hypothesis was tested that the government intervention resulted in a decrease in the incidence of DCI in the industry and an improved clinical outcome of divers with DCI.

Methods-The incidence of treated DCI in tuna farm divers was estimated from the number of divers with DCI treated and the number of dives undertaken extrapolated from a survey of the industry in 1997-8. General health was measured in the tuna farm diving population by a valid and reliable self assessment questionnaire. The outcome of the divers treated for DCI was analysed with a modified clinical severity scoring system.
\end{abstract}

Results-The apparent incidence of treated DCI has decreased in tuna farm divers since the government intervention. The evidence supports a truly decreased incidence rather than underreporting. The general health of the tuna farm divers was skewed towards the asymptomatic end of the range, although health scores indicative of DCI were reported after $1.7 \%$ of the dives that did not result in recognised DCI. The clinical outcome of the divers treated since the intervention has improved, possibly because of earlier recognition of the disease and hence less time spent diving while having DCI.

Conclusions-The government intervention in the tuna industry in South Australia has resulted in a reduced incidence of DCI in the industry.

(Occup Environ Med 2001;58:124-128)

Keywords: decompression illness; aquaculture; education, professional

A $67 \%$ reduction in the tuna catch quota in South Australia in the early 1990s prompted fishermen in the Port Lincoln area to develop tuna farming. The first trial farms were established in 1991 and now there are 14 individual farming operations running 60 pens. During summer, tuna are caught in the Great Australian Bight and herded into cages, which are then towed to Port Lincoln. The fish are then transferred to stationary pens and fed pilchards before being hand harvested for the Japanese sashimi market.

About 45 divers are employed full time, increasing to 70 during the catching season. They inspect, maintain, and repair cages and pontoons, remove dead fish and occasionally sharks from the enclosures, and monitor the herding, feeding, and hand harvesting of the tuna. Fishermen with little knowledge of occupational diving developed the initial farming procedures. Divers were recruited locally and they were usually recreationally trained. Diving equipment and practice were not of occupational standard. ${ }^{1}$

By 1995, WorkCover Corporation (South Australia) had received 39 diving related claims from this industry, $A \$ 600000$ had been paid in compensation, and 17 divers had been treated for decompression illness (DCI) at the Royal Adelaide Hospital. Many of these divers did not recover completely and compensation has since escalated to $\mathrm{A} \$ 1600000$. This is not surprising as even promptly treated DCI has a high risk of major sequelae. ${ }^{2}$ In response, the Department for Industrial Affairs (subsequently renamed Department for Administrative and Information Services) and WorkCover Corporation, with the cooperation of the Tuna Boat Owners Association, implemented strategies to raise the standard of diving to that recommended for occupational diving. ${ }^{1}$ After introduction of these measures, the occurrence of treated DCI decreased. ${ }^{3}$

It has been claimed that these interventions have improved the standard of tuna farm diving and that this is primarily manifest as a reduction in the number of such divers seeking treatment for DCI. ${ }^{3}$ However, an apparent reduction in incidence of DCI could be explained in two ways. Firstly, the number of DCI cases may have decreased as a result of reduced diving exposure. Secondly, the intervention may have created an environment in which divers would not report symptoms of DCI, or would only report severe symptoms of DCI. Clearly, before any benefit can be claimed for this intervention, further analysis is required. Real incidence data will resolve the first issue, whereas the question of changed reporting behaviours requires assessment of the ongoing health of divers working in tuna farms. With such data, we hypothesise that the government interventions has not only resulted in a decrease in the incidence of DCI in tuna farm divers, but also in improved awareness, so that divers are treated earlier and more successfully. 


\section{Methods}

ASSESSMENT OF THE INCIDENCE OF TREATED DCI AND HEALTH OF THE DIVING POPULATION As the Hyperbaric Medicine Unit at the Royal Adelaide Hospital is a unique local resource, it can be assumed that all tuna farm divers who sought definitive medical care for DCI were treated at this hospital. The number of divers treated for DCI need to be evaluated against total diving exposure, diving patterns, and general health of the tuna farm divers. The number of full time divers can be estimated from the Department for Industrial Affairs site audits. Before 1995, record keeping of the diving operations was poor and whereas record keeping has improved, an industry wide, complete audit of diving exposure was impractical. Reliable estimates are available for the years 1997 and 1998 when diving operations contributed diving logs to this laboratory for evaluation of diving patterns. As divers are used in all aspects of the tuna catching and farming operations, total diving exposure in previous years can be extrapolated from annual tuna catch data from the Tuna Boat Owners Association of Australia. General health has been measured by a valid, reliable self assessment questionnaire. ${ }^{4}$

CLINICAL PRESENTATIONS OF DIVERS WITH DCI Approval to review case notes was obtained from the Ethics Committee and Medical Staff Society, Royal Adelaide Hospital, and conducted in accordance with the National Health and Medical Research Council Statement on Human Experimentation and Supplementary Notes: 1992. Twenty two tuna farm divers were treated for DCI between August 1993 and January 1998. One diver was treated for two incidents of DCI. Twenty one case notes (22 incidents of DCI) could be located and were reviewed by one of us (PW). Only one of these divers was lost to follow up after the initial recompression treatments. All 232 clinical assessments of these divers were reviewed. Assessments included those before each recompression treatment, at discharge, at the first follow up (typically at 6 weeks), and yearly follow up for those with continued disease. Divers whose disease prevented return to diving at the last review were contacted by telephone to discuss aspects of their illness.

For inferential analysis, each assessment was scored. The conventional practice, whereby decompression illness is classified as mild (type 1) or severe (type 2) decompression sickness, or arterial gas embolism, and recovery is categorised as complete, incomplete, and nil, is not useful in this context. Several scoring systems for neurological signs of DCI have been developed ${ }^{5-7}$ but many important symptoms and signs are not scored, confounding factors are not taken into account, and they are not designed to track patients' progress. For this study we used a clinical scoring system that has recently been developed and validated. ${ }^{8}$ This system scores 24 signs and symptoms on a scale of $0-3$ (none, mild, moderate, or severe) based on objective findings for signs and semantic anchors for symptoms. Items scored by this system are listed in table 1 . Each item
Table 1 Signs and symptoms of decompression illness

\begin{tabular}{lccc}
\hline Signs and symptoms & $\begin{array}{c}\text { Any time } \\
(\%)\end{array}$ & $\begin{array}{l}\text { First } \\
(\%)\end{array}$ & $\begin{array}{l}\text { Final } \\
(\%)\end{array}$ \\
\hline Pain & 95 & 95 & 57 \\
Paraesthesiae & 91 & 59 & 24 \\
Lethargy & 82 & 77 & 29 \\
Cognitive change & 77 & 68 & 29 \\
Objective sensory change & 68 & 55 & 0 \\
Balance & 59 & 45 & 19 \\
Reflexes & 59 & 36 & 14 \\
Headache & 55 & 41 & 14 \\
Mood change & 50 & 27 & 29 \\
Upper limb coordination & 36 & 27 & 10 \\
Lower limb weakness & 32 & 27 & 5 \\
Upper limb weakness & 27 & 18 & 5 \\
Dizziness & 18 & 9 & 0 \\
Tinnitus & 14 & 9 & 5 \\
Urinary problems & 14 & 5 & 5 \\
Nausea & 9 & 5 & 0 \\
Lower limb coordination & 9 & 5 & 5 \\
Gait & 5 & 0 & 5
\end{tabular}

The percentage of tuna farm divers (22 incidents of decompression illness) with various symptoms and signs before their first hyperbaric treatment (presentation), at the final follow up assessment, and at any time. Also included in the clinical severity scoring system but not found in these divers are visual, hearing, or speech disturbances; lymphatic involvement; bowel dysfunction; and rash.

score is then weighted according to specificity for DCI, natural history if left untreated, potential to incapacitate, and codependent symptoms. Codependent symptoms or signs cannot be simultaneously assessed-for example, lower limb weakness will interfere with or prevent assessments of gait, balance, and coordination. Only the dominant symptom or sign is scored and weighted according to the number of codependent signs and symptoms. The sum of all item scores is used as an index of severity of disease.

The clinical scoring system was designed for prospective use and was revised here for retrospective use. Firstly, not all of the scored signs and symptoms were noted at each assessment; however, as the assessments were made by experienced diving medicine specialists it was assumed that they were not overlooked. Some signs and symptoms not specifically mentioned could be implied or interpolated from indications in preceding and subsequent assessments and from remarks such as "no change since the last assessment". There was a significant difference (paired $t$ test, two tailed $\mathrm{p}<0.001$, $\mathrm{df}=231$ ) between the summed severity scores obtained with the original and the revised system formats. Although the difference was clinically small (0.52 (1.65) points, mean (SD), $\mathrm{n}=232$ ) and strongly correlated (Pearson's $r=0.99$ ), the revised format was used as we thought it more accurately reflected the clinical situation.

Secondly, the original system weights (over the range $0.75-1.25$ ) the scores for each sign and symptom according to whether it is remitting, static, progressive, or relapsing. This evolution of symptoms could only be evaluated for 506 of the 1229 scored signs and symptoms $(41 \%)$. The value of weighting schemes in multi-item inventories has been questioned, ${ }^{10}$ particularly where the range of weights is small. Where available, item scores obtained with or without the weighting were not significantly different (paired $t$ test, two tailed $\mathrm{p}=0.753$, $\mathrm{df}=505)$ and were strongly correlated (Pear- 
Table 2 Clinical progress and delay to treatment

\begin{tabular}{lll}
\hline & Short delay & Long delay \\
\hline Presentation score & $19.9(11.2)^{\star} \ddagger$ & $23.2(14.9)^{\star} \$$ \\
Final score & $3.0(6.5) \dagger \ddagger$ & $14.6(17.6)+\$$ \\
Return to diving & $5 / 7 \Phi$ & $5 / 14 \uparrow$ \\
\hline
\end{tabular}

${ }^{\star} \mathrm{p}=0.601 ; \mathrm{tp}=0.111 ; \neq \mathrm{p}=0.003 ; \mathrm{g}=0.133 ; \uparrow \mathrm{p}=0.132$.

$\star+\nabla$ Compare horizontally; $\neq \$$ compare vertically.

Mean (SD) presentation scores and final scores (paired $t$ test, two tailed $\mathrm{p}$ values) for divers with short delay to treatment and divers who delayed treatment and continued diving with symptoms of decompression illness (long delay). Proportion of divers returning to diving ( $V^{2}$ test, two tailed $\mathrm{p}$ value). One diver lost to follow up is not included in the return to diving data.

son's $r=0.98)$. As the weighting scheme was redundant and data not always available, it was not used in this study.

DATA ANALYSIS

Results are presented as means (SDs) or occasionally mean (range). Clinical severity scores are tabulated (table 2) at presentation and at final assessment. The interval between presentation and final assessment differs for each diver. Comparisons between groups were made with unpaired $t$ tests for comparisons between groups and paired $t$ tests were used for comparisons within groups (two tailed $\alpha=0.05)$. Proportions were compared with the $V^{2}$ test, a modification of the $\chi^{2}$ test scaled for sample size (two tailed $\alpha=0.05$ ). Statistical analysis was performed with Statistics for Windows (Statsoft, Tulsa, OK, USA.). All data were collated and clinical scores assigned with a database application (Access 95, Microsoft, Redmond, WA, USA).

\section{Results}

INCIDENCE OF TREATED DCI IN TUNA FARM DIVERS

During 1998, 3572 tonnes of tuna were captured into the farms by about 48 full time divers. About 6000 dives (2000 hours) were conducted during this season. This ratio of fish caught to dives undertaken was used to extrapolate data for 1996 (recorded catch 3362 tonnes of tuna and 49 full time divers audited) and 1994 (recorded catch 1294 tonnes of tuna and 35 full time divers audited). During 1994 (before the intervention strategies), there were

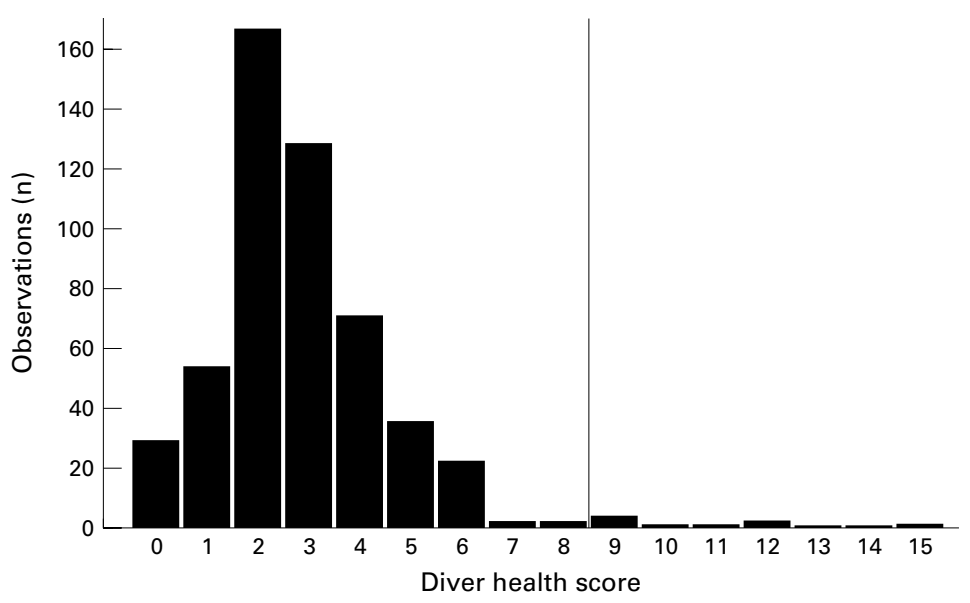

Figure 1 Frequency histogram of diver health scores for 517 days of diving. Validation of the diver health survey showed scores $\geqslant 8$ (vertical line) are indicative of possible decompression illness.
14 incidents of DCI from 2100 dives resulting in an estimated incidence of six DCIs/1000 dives (20/1000 hours). After the strategies, during 1996, there were three incidents of DCI from 5450 dives, resulting in an estimated incidence of 0.5 DCIs per 1000 dives (1.6/1000 hours). These incidences are significantly different $\left(V^{2}\right.$ test, two tailed $\left.\mathrm{p}=0.000\right)$. Diving procedures have become more efficient since the introduction of occupational diving standards so that the 1994 figures probably overestimate the incidence of DCI/1000 hours of diving exposure.

ONGOING HEALTH OF TUNA FARM DIVERS

Self assessment during 1997 and 1998 produced a distribution of diver health scores in the general tuna farm diving population skewed towards the asymptomatic end of the range (diver health score=0) (fig 1). After dives that did not result in retrieval to the Royal Adelaide Hospital, nine of 517 scores (1.7\%) were greater than the threshold indicative of DCI. ${ }^{4}$ Four divers returned these high scores. Three divers reported single, transient episodes of poor health with subsequent continued reports of good health. These seem unlikely to be DCI. One diver reported four high scores each followed by up to 5 months of good health reports.

CLINICAL PRESENTATIONS OF TUNA FARM DIVERS WITH DCI

All divers were male and the average age was 29 years (range 19-43). Divers received an average of eight recompression treatments (range 2-14). All divers received an initial United States Navy treatment table $6,{ }^{11}$ and subsequent treatment tables varied. The distribution of signs and symptoms among these divers, as scored in the clinical scoring system, is listed in table 1 . Seven divers were symptom free at discharge and all of these eventually returned to diving. Fifteen divers were discharged with residual symptoms, and 12 remain unwell and have not returned to diving. Three of the 12 divers were unable to return to any form of work because of severe residual symptoms.

A striking feature of these divers was that many continued diving while they had DCI. In 15 of the 22 incidents, divers continued to dive with symptoms of DCI before seeking treatment and mean delay to treatment was 72 days (range 14-270 days). For the remaining seven incidents of DCI, divers stopped diving and sought treatment so that the mean delay to treatment was 4 days (range 1-14 days). The delay to treatment was significantly different between these groups (unpaired $t$ test, two tailed $\mathrm{p}=0.016$ ). Table 2 summarises the clinical severity scores and outcomes of these two groups. No significant difference was found between mean presentation and final scores for these two groups. However, the short delay group made better recovery, as shown by a significant difference between their presentation and final scores. The divers who delayed treatment and continued to dive did not make a good recovery. Recovery was reflected in the 
Table 3 Decompression illness and government intervention

\begin{tabular}{lll}
\hline & Before intervention & After intervention \\
\hline Cases of decompression illness & 16 & 6 \\
Proportion diving when unwell & $13 / 16^{\star}$ & $2 / 6^{\star}$ \\
Delay in presentation (days) & $63(71) \dagger$ & $18(22) \dagger$ \\
\hline
\end{tabular}

${ }^{\star} \mathrm{p}=0.036 ; \mathrm{tp}=0.144$, before $v$ after intervention.

Incidents of decompression illness, the number of divers continuing to dive when unwell, and the delay to presentation before and after the beginning of the government intervention strategy in February 1995. Proportion of divers diving when unwell ( $V^{2}$ test, two tailed p value). Mean (SD) days to presentation (paired $t$ test, two tailed $\mathrm{p}$ values).

Table 4 Clinical progress and government intervention

\begin{tabular}{lll}
\hline & Before intervention & After intervention \\
\hline Presentation score & $24.2(14.0)^{\star} \ddagger$ & $16.7(12.1)^{\star} \Phi$ \\
Final score & $13.4(17.6) \dagger \ddagger$ & $4.2(6.8)+\$$ \\
Return to diving & $7 / 16 \uparrow$ & $3 / 5 \Phi$ \\
\hline
\end{tabular}

${ }^{\star} \mathrm{p}=0.260 ; \mathrm{tp}=0.232 ; \neq \mathrm{p}=0.067 ; \mathrm{g} \mathrm{p}=0.022 ; \uparrow \mathrm{p}=0.535$. $\star+\uparrow$ Compare horizontally; $\ddagger \S$ compare vertically.

Mean (SD) presentation scores and final scores (paired $t$ test, two tailed $p$ values) before and after the beginning of government intervention strategy in February 1995. Proportion of divers returning to diving ( $V^{2}$ test, two tailed $\mathrm{p}$ value). One diver lost to follow up is not included in the return to diving data.

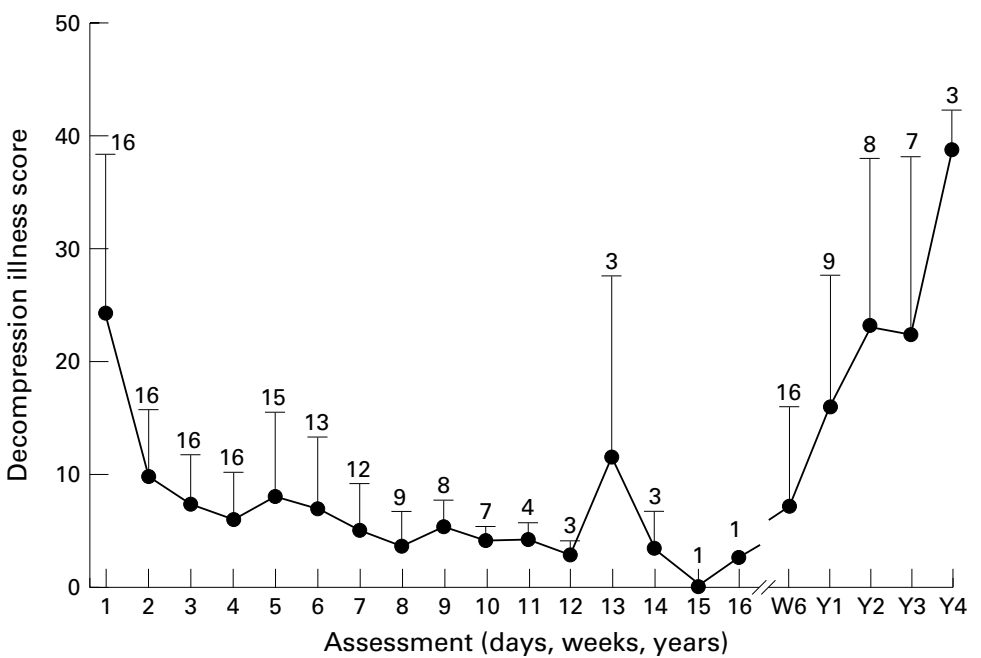

Figure 2 Mean severity scores and assessments for DCI before the government intervention. Mean DCI severity score for the divers before each recompression therapy (treatment numbers before break in $x$ axis) and long term progress (follow up assessments at 6 weeks (W6) and years 1-4 (Y1-Y4) after break in $x$ axis). Error bars are one SD and the number of divers assessed on each occasion is indicated above the bars. Note that half of the divers received nine or more recompression treatments.

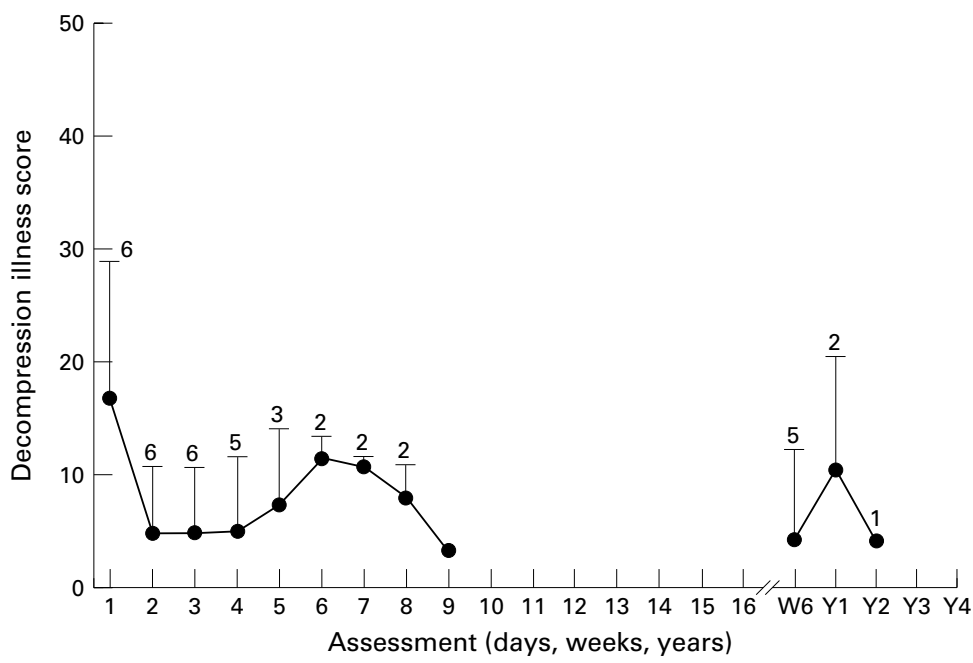

Figure 3 Mean severity scores and assessments for DCI after the government intervention. Axes and plot are the same as in figure 2. Note that these divers received many less recompression treatments than did the divers before the strategies. larger proportion of divers who returned to work after the shorter delay to treatment compared with the group who delayed treatment, although this was not significant.

\section{GOVERNMENT INTERVENTION}

Before the intervention by the Department for Industrial Affairs and WorkCover Corporation 16 divers presented for treatment for DCI (August 1993 to January 1995). At the time of this review six divers had presented for treatment since the government intervention (February 1996 to February 1998). As well as the reduced incidence of DCI reported here, one notable change in diving practice after the government intervention was a reduction in the number of divers who continued to dive with symptoms of DCI (table 3). There was considerable variability in the delay to presentation in both groups and although there was an apparent decrease in days to presentation after the intervention, this did not reach significance. In the group diving before intervention four divers waited 3-9 months to seek treatment. In the group who dived after intervention the longest delay to treatment was 2 months.

The clinical severity scores and outcomes between divers presenting before and after the government interventions are shown in table 4 . There was no significant difference in the mean severity of scores either on presentation or at last review between these groups. However, there is some evidence of better recovery in divers presenting with DCI after the intervention, as there was a significant difference between their presentation and final scores. By contrast, the difference between final and presentation scores of divers presenting before the intervention did not reach significance. A greater proportion of divers who presented after than before intervention returned to diving, but this did not reach significance.

The progression of the disease severity index during treatment and at subsequent follow up for the divers, both before and after the government intervention, is shown in figures 2 and 3. Before the intervention divers improved during treatment but many deteriorated during the subsequent years. After the intervention divers generally continued to improve.

\section{Discussion}

These data suggest that the diving practice associated with tuna farms in South Australia has been positively reformed after a State Government intervention. The 1 year (1995) accident reduction strategy was to apply, where possible, standard occupational diving practice ${ }^{1}$ to tuna farm diving. This included occupational training of divers. The intervention comprised four on site audits, consequent issue of improvement and prohibition notices, training sessions, and assistance with development of an industry diving operations manual. Annual on site audits and continued Government contribution to diver training followed this strategy. The approved code of practice for tuna farm diving was gazetted on 24 March 1997.

Government review of their strategy used diver training as an index of compliance in the 
industry and cost of compensation as an index of severity of injury. ${ }^{3}$ In the 2 years before the accident reduction strategy $47 \%$ of divers in the industry had occupational training, WorkCover Corporation had received 39 diving related claims from this industry at a cost of $\mathrm{A} \$ 1600$ 000, and 17 divers had been treated for DCI. In 4 years since the intervention occupational trained divers comprised $100 \%$ of the industry, there have been a further 24 compensation claims at a cost of $\mathrm{A} \$ 55000$ and six divers have been treated for DCI.

The claimed reduction in number of cases of DCI in tuna farm divers is reinforced here by a reduction in incidence of divers being treated at the Royal Adelaide Hospital. However, this change in incidence may be the result of altered reporting. Current health self assessment indicates possible underreporting of DCI and the extent of unreported DCI before the intervention is unknown. However, $1.7 \%$ may be a low frequency of unreported DCI for a fishing industry. In one survey, Australian abalone divers only sought definitive treatment for $15 \%$ of incidents of DCI. ${ }^{12}$ Also, the occupational diving standard ${ }^{1}$ includes a formal system of accident reporting and an annual medical examination for divers. These facts do not support an increase in unreported DCI.

Whereas a zero incidence of DCI is impossible for any diving operation, incidents in this industry still require further investigation. Given this low current overall risk of DCI and the skew of the health status scores, it is possible that specific high risk factors may have operated in a few dives to result in DCI. It will be important to identify any characteristic features of the dives that resulted in either DCI treated at the Royal Adelaide Hospital or in health scores greater than 8 , so that corrective strategies can be developed or maintained.

The likelihood of a positive reform of diving practice here is also supported by the finding that significantly fewer divers with DCI continued to dive after the intervention. Not surpris- ingly, given that delay to presentation and continuing to dive after the development of DCI are significant risk factors for poor outcome, ${ }^{2}$ the divers with DCI after the intervention tend towards a better response to treatment and return to diving.

Aquaculture is a growth industry world wide. Legislation to regulate diving practice associated with fish farming elsewhere has not been objectively audited for efficacy. By several objective measures, the strategy of the Government to reduce accidents in tuna farm diving has reduced risk in this industry. Decompression illness has a high risk of sequelae ${ }^{2}$ and any intervention that reduces risk in this context should be supported.

This work was supported by a grant from WorkCover Corporation, South Australia.

1 Standards Australia Occupational diving. Australian standard. Sydney: Standards Australia, 1992. (AS 2299-1992.)

2 Moon RE, Gorman DF. Treatment of the decompression disorders. In: Bennett PB, Elliott, DH, eds. The physiology and medicine of diving, 4 ed. London: WB Saunders, 1993.

3 Craig DS. Aqua culture (tuna farms) diving safety. Government report. Adelaide, South Australia: Workplace Services, Department for Administrative and Information Services, 1998.

4 Doolette DJ. Psychometric testing of a health survey for field reporting of decompression outcome. Undersea Hyperb Med 2000 (in press)

5 Dick AP, Massey EW. Neurologic presentation of decompression sickness and air embolism in sport divers. Neurology 1985;35:667-71.

6 Kelleher PC, Pethybridge RJ, Francis TJ. Outcome of neurological decompression illness: development of a manifestation-based model [comments]. Published erratum in Aviat Space Environ Med 1997;68:246. Aviat Space Environ Med 1996;67:654-8.

7 Boussuges A, Thirion X, Blanc P, et al. Neurologic decompression ill

8 Mitchell SJ, Holley T, Gorman DF. A new system for scoring severity and measuring recovery in decompression ing severity and measuring recovery in decompression 1999;28:84-94.

9 Holley T. Validation of the RNZN system for scoring severity and measuring recovery in decompression illness. South Pacific Underwater Medicine Society fournal 2000;30:75-80.

10 Nunnally JC. Psychometric theory, 2 ed. New York: McGrawHill, 1978.

11 US Navy diving manual (air diving), revision 1 ed. Washington, DC: Naval Sea Systems Command, 1985.

12 Andrews G, Holt P, Edmonds C, et al. Does non-clinical decompression stress lead to brain damage in abalone divers? Med f A ust 1986;144:399-401. 